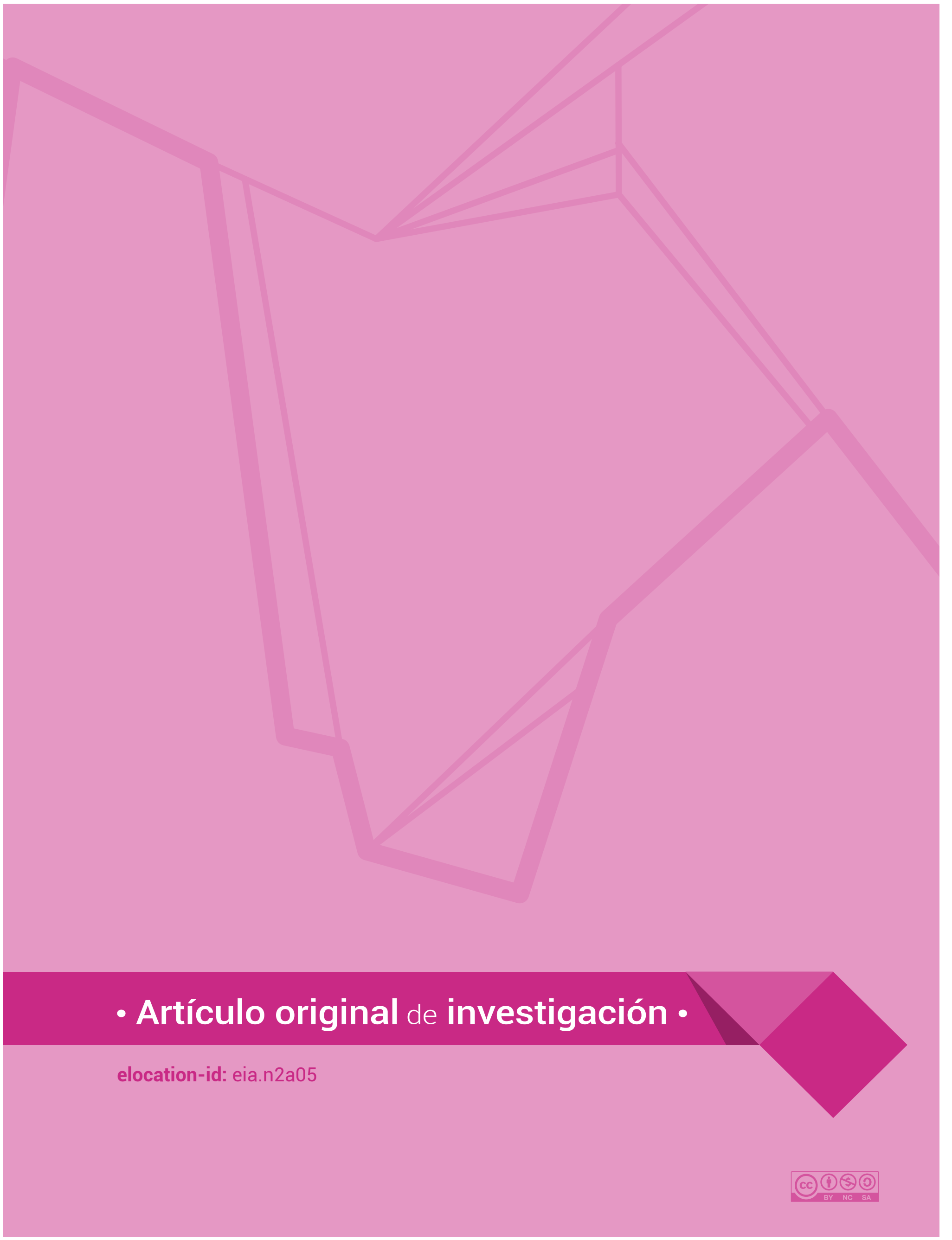




\title{
Análisis en las instituciones de educación básica y media de Manizales frente a las necesidades de formación en normas internacionales de contabilidad en el sector público para el año 2016
}

\author{
Analysis of the training needs associated to \\ the International Public Sector Accounting \\ Standards in institutions of basic and high \\ school education of Manizales for the year 2016
}

María Natalia Ortega-Aguilar', Luz Adriana Sepúlveda Pérez², Luz Elena Muñoz Sánchez ${ }^{3}$

Especialistas en Revisoría Fiscal y Contraloría, Facultad de Ciencias Contables, Corporación Universitaria Remington, Manizales - Caldas, Colombia.

1 nataliaortagu@yahoo.es

2 adriana.sepulveda20@hotmail.com

3

Correo electrónico: todoseguros777@gmail.com

\section{Resumen}

El direccionamiento del proyecto de investigación está enfocado a la descripción del marco normativo aplicable a entidades de gobierno del sector educativo del régimen de contabilidad pública, teniendo en cuenta que, Colombia es uno de los países que se encuentra en proceso de aplicación plena para todos sus sectores económicos, y en ese proceso ha incorporado las normas internacionales de contabilidad en el sector público (NICSP) dentro del régimen de contabilidad pública. No obstante, la puesta en marcha de la nueva normativa aún tiene un vacío y es la guía para el proceso de implementación de todos los cambios. De acuerdo con la Contraloría General de la Nación, se deben diseñar planes de acción para ese proceso, los cuales están a cargo de las altas directivas. Sin embargo, dichos planes aún no existen y el principal problema es que la puesta en ejecución de la norma empieza para la vigencia 2017; bajo este contexto, el presente artículo busca abordar un elemento importante dentro del plan de acción, y es la capacitación del personal, logrando caracterizar las necesidades de formación en términos contables, para que se pueda realizar efectivamente el proceso de implementación de la norma. Dado el amplio número de entidades estatales, se decidió tomar como objeto de estudio práctico 35 instituciones públicas de educación básica y media de la ciudad de Manizales. Por tanto, se logra identificar las características 
del perfil actual del personal contable, sus necesidades de formación y las actualizaciones que se deben hacer al perfil de este personal para que a futuro todos los funcionarios estén en capacidad de cumplir con los requerimientos de la norma. Los resultados se consideran un aporte en la construcción de los planes de implementación de las NICSP y, por supuesto, permitirán que el ente objeto de estudio avance más rápidamente en dicho proceso.

Palabras claves: contabilidad, Estado, IFAC, marco normativo, regulación, transición.

\section{Abstract:}

The research project is focused on the description of the public accounting regulatory framework applicable to government entities of the education sector. Colombia is in the process of fully applying this regulatory framework to all of economic sectors, and as part of that process, it has introduced the International Public Sector Accounting Standards (IPSAS) to the public accounting system. However, the implementation of new regulations still lacks a guide for the process to implement all changes. According to the Office of the Comptroller General of Colombia, action plans must be designed for this process by senior officers. Such plans have not been yet developed and the main problem is that the implementation of the standard begins for 2017. Against this backdrop, this paper aims to address an important element in the action plan, the training of staff. It seeks to characterize the accounting training needs which are paramount to effectively implement the standards. Given the large number of state agencies, 35 public institutions of elementary and high school education of the city of Manizales were chosen as objects of study. As a result of this analysis, it was possible to identify the characteristics of the current profile of accounting personnel, their training needs and the updates that should be made to their profile so that in the future all officers can comply with the standard requirements.
These results are considered as a contribution to the design of the implementation plans of IPSAS and will certainly allow the entity under study to advance more quickly in this process.

Keywords: accounting, State, IFAC, regulatory framework, regulation, transition.

\section{Introducción}

La Contaduría General de la Nación (CGN), como ente regulador de la contabilidad de todas las instituciones del Estado y como autoridad en materia de normatividad contable, viene adelantando un proceso de modernización para centralizar, consolidar y dar uniformidad a la contabilidad pública, de manera que sea posible elaborar un balance general de las cuentas del Estado, que sirva como soporte en la toma de decisiones para la administración pública y contribuya a una gestión del Estado más eficiente y transparente.

Dentro de dicha modernización la CGN ha decidido hacer una adopción indirecta de las NICSP, de acuerdo con la Federación Internacional de Contadores (IFAC - International Federation of Accountants) (2009):

Las NICSP establecen los requisitos para la elaboración de informes financieros que emiten los gobiernos y otras entidades del sector público que no sean empresas comerciales del gobierno. Las NICSP sobre el método contable de acumulación se basan en las Normas Internacionales de Información Financiera [NIIF] que son emitidas por el Consejo de Normas Internacionales de Contabilidad (International Accounting Standards Board/IASB) en la medida en que los requisitos incluidos en esas normas se apliquen al sector público (párr. 2). 
De esta manera la CGN planteó la definición de un marco normativo que cumple las siguientes cuatro características:

- Tomar como referente las NICSP.

- Privilegiar criterios de reconocimiento, medición, relevancia y presentación de informes.

- Homogenizar políticas contables.

- Definir criterios técnicos contables aplicables al sector gobierno.

Todo lo anterior quedó señalado en la Resolución 533 (2015b) de la CGN, por la cual se incorporó el marco normativo aplicable a entidades de gobierno dentro del régimen de contabilidad pública, estableciendo un periodo de preparación obligatorio y un primer periodo de aplicación. El periodo de preparación está comprendido desde la publicación de la resolución hasta el 31 de diciembre de 2016 y sugiere la elaboración de un plan de acción relacionado con la elaboración obligatoria del marco normativo. El primer periodo de aplicación corresponde a todo el año 2017 y en él, las entidades definirán los activos, pasivos, patrimonio y cuentas de orden, de acuerdo con los criterios del nuevo marco.

El presente artículo está asociado principalmente con el periodo de preparación y con los planes de acción que debe formular la alta dirección para realizar la implementación del marco normativo. De modo general, el objetivo de los planes de acción debe ser apoyar estratégica y operativamente la implementación institucional de las NICSP en cada una de las entidades estatales, estableciendo acciones, tareas y actividades que aseguren un adecuado cumplimiento de la transición. Dentro de los planes de acción se contempla el análisis de las normas contables que le serán aplicadas a las instituciones, la identificación de necesidades de capacitación y la evaluación del impacto sobre sus procesos, sistemas y cultura organizacional.
Dado que actualmente los planes para la implementación de las NICSP en las entidades públicas de Colombia solo se han centrado en los criterios del marco normativo, tal cual lo manifiesta la resolución 414 (2014b) de la CGN y sus modificaciones; la presente investigación está interesada en abordar el desarrollo de los planes de acción de la implementación, centrándose en una de las primeras etapas de dicho proceso, concerniente a la gestión del conocimiento, la cual es vital para el desarrollo del mismo. Normalmente la gestión del conocimiento comprende tres acciones: capacitación, aprendizaje compartido y experiencia. En este caso, el presente artículo se centra en la capacitación.

Cualquier proyecto de implementación requiere de procesos de formación continua a diferentes niveles y orientada a diferentes grupos. En tal caso, es necesario realizar una segmentación de las necesidades de capacitación con el fin de cerrar las brechas existentes entre la actual estructura y el nuevo marco normativo. En tal sentido es indispensable caracterizar los requerimientos del personal contable por segmentos (responsabilidad, funciones, perfil profesional) para fortalecer las competencias necesarias de cada función (Contraloría General de la República de Chile, 2015, p. 10).

En este sentido, como ya lo había anticipado la CGN (2013), la implementación de las NICSP requiere un alto componente de capacitación tanto para la socialización como para la asimilación del nuevo marco normativo. Esto implica que los procesos de capacitación deben contar con personal suficiente y calificado que tenga conocimiento en los modelos internacionales de contabilidad y en el sector público colombiano. Esto con el fin de apoyar a funcionarios en los procesos de centralización y consolidación de la Información de la CGN. Por tanto, se prevé que en el corto plazo haya un incremento 
drástico en el volumen de consultas y de solicitudes de capacitación, debido a las dificultades que se puedan presentar en la interpretación del nuevo marco regulatorio. Este impacto puede resultar permanente por la alta rotación de los responsables de la información financiera, especialmente, en las entidades territoriales.

Teniendo en cuenta lo anterior, esta investigación tuvo como objeto práctico de estudio las instituciones públicas de educación básica y media de la ciudad de Manizales, con el fin de caracterizar las necesidades de formación de su personal contable para la implementación de las NICSP. Este tipo de instituciones se caracterizan por tener un manejo descentralizado de fondos, pero cuya información se debe administrar por el ente territorial a cargo, el cual es la Alcaldía Municipal. En el caso de los fondos, su ejecución está sujeta a la normativa impuesta en el decreto 4791 (2008) del Ministerio de Educación Nacional y es deber de los rectores presentar los reportes de información financiera de acuerdo con la normativa existente y en los plazos establecidos por los organismos de control y la CGN.

Por tanto, dado que la ejecución de fondos en estas instituciones es una actividad constante durante toda la vigencia anual y que los informes hacia el ente territorial deben ser uniformes para facilitar la centralización de la información de acuerdo con la nueva normativa, es de esperarse que el perfil de los funcionarios a cargo de dicho proceso de ejecución se ajuste al nivel profesional requerido y al conocimiento y dominio de las competencias que le permitan desarrollar adecuadamente su labor.

Es por eso que el proceso investigativo buscó indagar sobre las características del perfil actual del personal contable en las instituciones públicas de educación básica y media de
Manizales, para caracterizar las necesidades de formación existentes para la implementación de las NICSP y así mismo sugerir las modificaciones que se debe hacer al perfil de este personal para que a futuro todos los funcionarios estén en capacidad de cumplir con los requerimientos de la norma.

Se espera, por tanto, que los resultados de dicho estudio sean referentes en la generación de los planes de acción requeridos por cada institución del Estado para la implementación de las NICSP, al igual que ayude en este proceso a la entidad territorial objeto de estudio práctico, al formular un plan de capacitación acorde con las necesidades de su personal contable.

\section{Metodología}

\section{Tipo de Investigación}

Enfoque cualitativo-cuantitativo, fundamentado en el paradigma deductivo, con un diseño no experimental de tipo descriptivo, de corte transversal; dado que se consideró importante conocer cuál era el comportamiento de algunas variables, como lo es el perfil del personal encargado del área contable en las instituciones públicas de educación básica y media de la ciudad de Manizales y de esta forma evaluar la implementación de las NICSP como situación específica para el año 2016 (Hernández, Fernández \& Baptista 2010).

Se tuvo el siguiente proceso de recolección de la información: primero se efectuó una revisión de los documentos referentes a las NICSP para determinar los cambios que se exigían sobre esta en relación al régimen de contabilidad pública (RGP), para su implementación en las entidades públicas en general. Segundo se 
establecieron cuáles de los cambios identificados sobre el actual Régimen de Contabilidad Pública para la implementación de las NICSP aplicaban y tenían mayor incidencia sobre las instituciones de educación pública.

Tabla 1. Instrumento de recolección de la información.

\begin{tabular}{|c|c|c|}
\hline \multicolumn{3}{|c|}{ Instrumento de recolección de la información } \\
\hline Tipo de instrumento & Encuesta & \\
\hline Número de preguntas & 23 & \\
\hline Tipo de preguntas & $\begin{array}{l}10 \\
7 \\
6\end{array}$ & $\begin{array}{l}\text { Dicotómicas } \\
\text { cerradas } \\
\text { Polítómicas } \\
\text { opción única } \\
\text { Abiertas }\end{array}$ \\
\hline Instituciones educativas & 72 & \\
\hline Encuestas enviadas & 72 & \\
\hline Encuestas contestadas & 35 & \\
\hline Encuestas analizadas & 35 & \\
\hline
\end{tabular}

Se aplicó un instrumento de recolección de datos (encuesta) (Tabla 1), para conocer el perfil actual del personal contable de las 72 instituciones públicas de educación básica y media de la ciudad de Manizales. La muestra neta fue de 35 instituciones públicas, que es el total de encuestas contestadas, equivalente al $48.6 \%$ de la población total. El instrumento fue analizado para identificar parámetros como nivel educativo, tipo de formación, experiencia en el cargo y por último se aplicó un instrumento de recolección de datos para identificar cuáles de los conocimientos y capacidades extraídas en la etapa 2 de la metodología, no estaban satisfechos por las capacidades de la actual planta de personal contable de las 72 instituciones públicas de educación básica y media de Manizales para llevar a cabo la implementación de las NICSP.

\section{Resultados}

\section{Sistema contable de las Instituciones públicas de educación básica y media de Colombia}

En el marco de la globalización los procesos macroeconómicos han cobrado un papel fundamental en el crecimiento de las economías, las unificaciones de modelos económicos han facilitado el poder de estandarizar políticas que permiten a las empresas participar en un mercado global, rompiendo los esquemas locales y siendo actores importantes en el contexto internacional. En este escenario no solo el sector privado ha jugado un papel fundamental, el sector público y/o sector gobierno también se ha convertido en actor de primera línea en la medida en que son los gobiernos junto con los organismos internacionales, los que articuladamente fijan y establecen las políticas en materia de estandarización de los procesos a nivel mundial.

En tal sentido, los entes territoriales, como parte del sector gobierno y de manera más particular los fondos de servicios educativos, cobran un papel fundamental en las políticas del Estado y deben cumplir, al igual que los demás estamentos gubernamentales, con los procesos de internacionalización y unificación de los procesos; las NICSP al igual que las NIIF en el sector privado ofrecen como mecanismo de estandarización la unificación de criterios para facilitar la incorporación de las entidades a las dinámicas financieras del sector, es así como y de manera particular los fondos de servicios educativos deben construir unas políticas contables claras que son implícitas para los estándares NICSP en aras de poder transitar la implementación en el año 2017. 
La Figura 1 muestra un resumen del manejo de la información contable en las entidades de carácter público, evidenciando los organismos de control, las funciones del ministerio de Hacienda y la información financiera que se debe presentar de acuerdo con sus periodos.

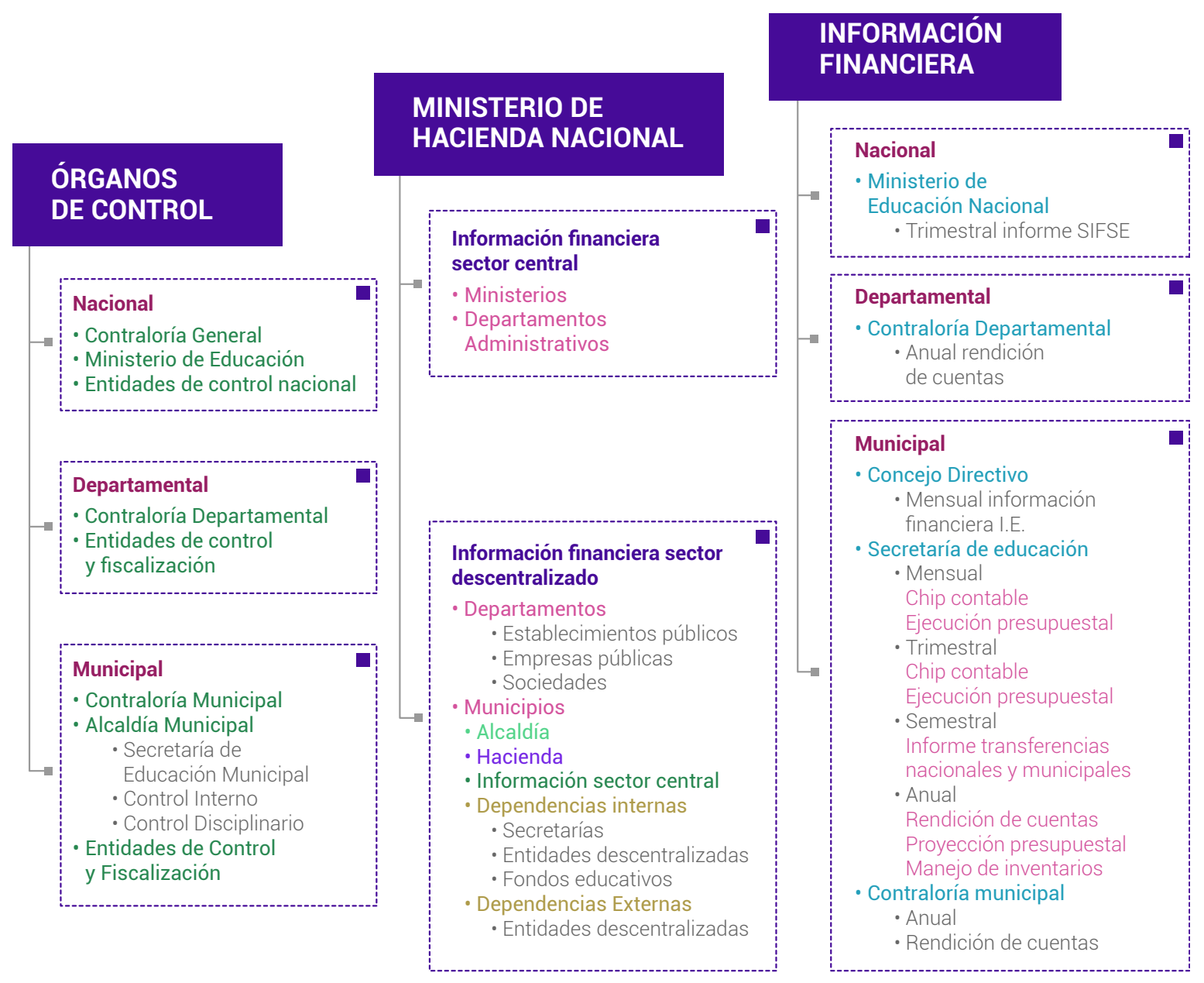

Figura 1. Manejo de la información contable.

Con respecto a la forma en que deben proceder las instituciones públicas de educación básica y media de Colombia para el manejo contable, proceso de tesorería, teneduría de inventarios y ejecución presupuestal de ingresos y gastos, es necesario precisar que son instituciones públicas que pertenecen al orden descentralizado del respectivo ente territorial y que por ello el sistema financiero se encuentra normatizado por las directrices que expide el Ministerio de Educación Nacional, la CGN, CGR, los pronunciamientos que emita el Ministerio de Hacienda y Crédito Público y los cambios normativos que emita el ente territorial. 
Es necesario enfatizar que es responsabilidad del ente territorial certificado, administrar el servicio educativo, al mismo tiempo debe organizar, planificar y distribuir los recursos económicos de cada fondo. Para ello cada institución educativa debe acreditar y adjuntar: licencia de funcionamiento, conformación del consejo directivo, consejo de padres, resolución de costos educativos, apertura de cuenta corriente denominada Fondo de Servicios Educativos con registro en la tesorería del ente territorial. Posteriormente y por medio de la secretaría de educación certificada y de acuerdo con el Decreto 4791 de 2008, se autoriza al consejo directivo y rector de cada establecimiento, para que elabore y presente el proyecto anual de presupuesto, el plan de compras y administre los recursos que el fondo perciba por la prestación de servicio, donaciones y transferencias de orden nacional, departamental o municipal; al cumplimiento de lo requerido por la dependencia encargada se procederá al funcionamiento de cada fondo.

Para dar inicio al proceso presupuestal y contractual cada institución educativa debe contar con un manual general que describa de manera detallada el marco legal aplicable a los fondos, seguido de los pasos que se deben adelantar para el recaudo, gasto, caja menor, sistema de contratación, manejo de inventarios, custodia de bienes y mecanismos de control interno; de igual manera y acorde a lo expuesto anteriormente, cada institución realizará el registro de los siguientes libros contables:

- Registro de ingresos.

- Registro de apropiaciones, compromisos, obligaciones y pagos.

- Registro de reservas.

- Mayor y balance (activos, pasivos y patrimonio) de acuerdo con el plan contable vigente.
- Libros auxiliares (comprobantes) de acuerdo con el plan contable vigente.

- Libro de inventarios.

- Libro de actas.

Cada libro debe estar debidamente foliado y diligenciado sin enmendaduras. Dado que el sistema financiero público tiene un tratamiento diferente al realizado por el sector privado, es necesario precisar que las entidades públicas plasman sus recursos económicos en el estudio de necesidades; para ser desglosados en el proyecto presupuestal, seguido de la aprobación que es presentada a la secretaria de educación certificada, para que a través de ella sea incorporado al presupuesto del ente territorial y de manera paralela incorporado al presupuesto del Ministerio de Hacienda Nacional.

Dando continuidad al sistema financiero contable conforme al Decreto 4791 de 2008, los fondos educativos deben llevar contabilidad de manera relevante, comprensible, fiable y verificable, que permita evidenciar de forma clara los hechos económicos. Simultáneamente el ente territorial por intermedio de la secretaria de educación certificada, fijará el cronograma y periodicidad para la entrega de los informes requeridos por los siguientes entes de control y vigilancia:

- Comunidad educativa - Informe de gestión

$$
\text { - Anual. }
$$

- Consejo Directivo - Ejecución Presupuestal, Informe de Contratación, Relación de Ingresos y Gastos, Propuestas Económicas, Ejecución Plan de Compras y Estados Financieros -mensual-.

- Secretaría de Educación Certificada - Chip Contable y Ejecución Presupuestal de Ingresos y Gastos - mensual y consolidada trimestral - Informe de Transferencias 


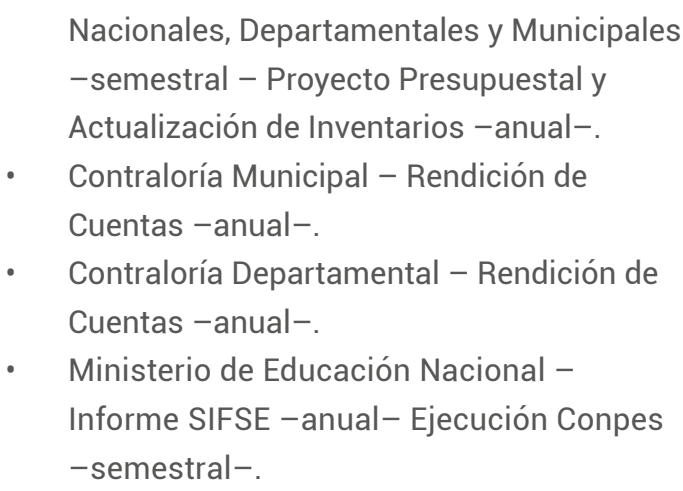

Los entes de control son encargados de vigilar el uso adecuado y destinación de los recursos percibidos por los fondos educativos, además, la ley los autoriza para realizar auditorías que consideren necesarias con el fin de verificar la veracidad, cumplimiento y tratamiento de la información reportada, al igual que los archivos que reposan de los estados financieros de la institución educativa.

En la actualidad, los fondos de servicios educativos manejan sus inventarios de bienes muebles con base en las directrices fijadas por la Contaduría General de la Nación, las mismas establecen, entre otras, que los inventarios de bienes muebles tienen dos clasificaciones. Una línea la componen todos aquellos elementos que pertenecen al ente central y que han sido donados por el municipio, aquellos que han remitido al Ministerio de Educación y todos lo que se han originado producto de convenios interadministrativos firmados por la Administración Municipal y la Academia, entidades sin ánimo de lucro, o de carácter especial como el Comité de Cafeteros y la fundación Luker. De otro lado, los bienes adquiridos con recursos propios que tienen como fuente de financiación los ingresos operacionales y las transferencias del sistema general de participación para la gratuidad de la educación.
Estos bienes, además de tener un origen específico, deben cumplir con unos lineamientos contables que facilitan su control. En el caso de los inventarios entregados y de propiedad del municipio cuyo manejo no es comodato, en la actualidad se registran en cuentas de orden, para que sean visibles en la contabilidad; los bienes de la institución se incorporan en la propiedad planta y equipo como parte de los activos fijos, teniendo que ser depreciados para establecer el gasto normal por su uso y desgaste en el tiempo.

Con la implementación de las normas internacionales aplicadas a los fondos de servicios educativos se prevé un cambio sustancial en la dinámica de los inventarios de bienes muebles, lineamientos que están consagrados en las políticas que se fijan y que tienen como campo principal de aplicación el manejo de los inventarios de bienes. En tal sentido, dichas políticas deben estar encaminadas a revaluar el principio de manejo de los bienes que son de propiedad del municipio y que pasarían según directrices del ente central a ser parte de la propiedad planta y equipo del establecimiento educativo.

Esta situación implica que los valores registrados en las cuentas de orden deberán ser trasladados a la cuenta 16 del activo, a una cuenta especifica denominada bienes recibidos de terceros sin contraprestación, lo anterior no significa bienes recibidos en comodato, sino bienes cuya tenencia y usufructo son de manejo y aprovechamiento solo por la institución educativa, lo que implica, además, que su depreciación deberá hacer parte de las dinámicas contables que maneje la institución. Es claro que este lineamiento apunta a que el control y el manejo de su desgaste por el uso brindado estén en manos de quien realmente se está beneficiando y de profesionales que conozcan la dinámica. 
Otra de las políticas, que se deberá adoptar con la implementación de las normas internacionales para el sector educativo, hace referencia a la activación de bienes que presenten una depreciación cero, es decir, que su valor por uso y desgaste impliquen una depreciación total por el tiempo; en tal sentido, la política se encamina a establecer como criterio el hecho de que aquellos bienes por su estado o condiciones al momento de aplicar la norma reflejen un mayor tiempo de vida útil, deberán ser valorizados y actualizado su valor en la contabilidad, lo que implicaría por consiguiente activar su respectiva depreciación.

En este propósito los Fondos de Servicios Educativos deberán considerar si el estado óptimo del bien permite establecer un número determinado de años más para uso del mismo, a fin de fijar los criterios que llevarán a determinar su depreciación. Esto implica por supuesto establecer un valor real del bien al momento de implementar dicha medida, valor que deberá ser determinado mediante avaluó por parte de un experto evaluador.

Lo anterior traerá consigo un incremento de la propiedad planta y equipo de la institución educativa, lo que se reflejará en un incremento del activo y por consiguiente del patrimonio fiscal. El costo histórico del bien se afectará en la medida que, por razones del avalúo, se actualizará a valores presentes de mercado entendiendo, claro está, el estado y las condiciones del mismo. Este procedimiento por supuesto trae consigo que la institución incurra en un gasto adicional, debiendo contratar peritos evaluadores en procura de actualizar los valores de sus inventarios a la realidad corriente que reclama el contexto y a la adopción de políticas propias de las normas internacionales en el sector público.
Finalmente, el impacto de la adopción de políticas claras y determinadas por la implementación de las normas internacionales en las instituciones educativas, si bien generará cambios circunstanciales en el manejo de los inventarios de bienes muebles y en la dinámica de su control, traerá consigo cambios importantes en la contabilidad; esto por razones del registro de los movimientos y operaciones en las cuales se incurra cuando entren en vigencia las políticas fijadas por la administración central.

El otro aspecto importante a resaltar es la realidad de la aplicación en las instituciones educativas, donde se evidencia que los procesos son realizados por auxiliares que en la mayoría de los casos son bachilleres con algunos cursos en el área contable, estas personas están facultadas para ejercer el control y la afectación a los procesos contables y financieros; es importante clarificar que la Contaduría General de la nación tiene por premisa que el proceso contable debe estar en cabeza de un profesional del área contable.

Durante el trabajo de campo es importante aclarar que de las 72 instituciones educativas que existen en la ciudad de Manizales, de acuerdo con los datos obtenidos por la Secretaría de Educación Municipal, solo 35 de estas participaron, convirtiéndose en nuestra nueva muestra para la investigación. A continuación, se presentarán los resultados más relevantes, de acuerdo con el análisis de las variables, dando lugar al cumplimiento del objetivo del instrumento de recolección de la información.

En la caracterización de los encuestados se pudo analizar que el $54 \%$ de los funcionarios son mayores de 45 años, seguido del $37 \%$ que se encuentran entre el rango de 36 a 45 años de edad y el $9 \%$ restante se encuentran entre 18 y 35 años. 
El $50 \%$ de los funcionarios públicos se encuentran en los cargos relacionados con la tesorería y el otro $50 \%$, de acuerdo con lo analizado, cumple doble función tanto de tesorería como la de rectores de las instituciones educativas. En cuanto a su grado de escolaridad el $32 \%$ de los funcionarios son profesionales, el $24 \%$ tienen maestría, el $12 \%$ cuentan con alguna especialización; el $9 \%$ por su parte cuenta con doctorado, el $18 \%$ son técnicos y/o tecnólogos respectivamente, y solo el $6 \%$ restante son bachilleres. Dentro de las profesiones analizadas de las 35 instituciones, solo 6 de las personas encuestadas son contadores públicos, los otros son auxiliares y tienen carreras no afines a las funciones de tesorería, lo que pone en el centro de la discusión a los funcionarios públicos encargados de la convergencia de las NICSP en las Instituciones educativas, pues no cumplen con los lineamientos que el Instituto Nacional del Contadores Públicos exige.

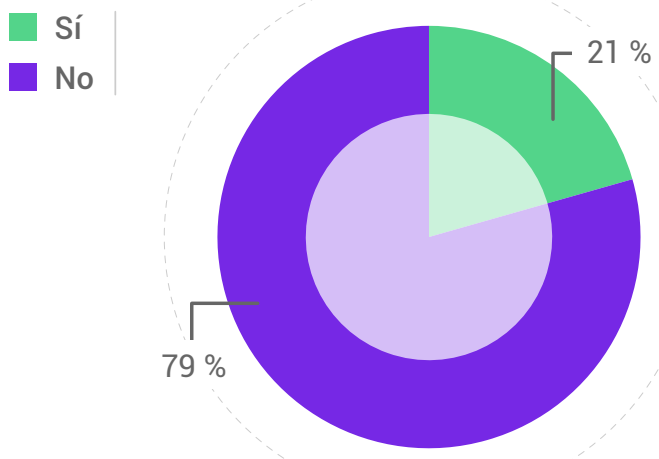

Figura 2. Conocimientos de los funcionarios de los colegios que conocen el plan de implementación de las NICSP.

El $79 \%$ de los encuestados no conocen la Resolución nro. 533 de 2015 que es el Plan de implementación, de acuerdo con las directrices de la Contaduría General de la Nación, ni el plazo para ejecutar las NICSP en su lugar de trabajo; solo el 21 \% afirma conocer el plan de implementación de las NICSP (Figura 2). 


\section{Capacitarse}

Conocer la Norma de las NICSP

Todas las anteriores

NS/NC

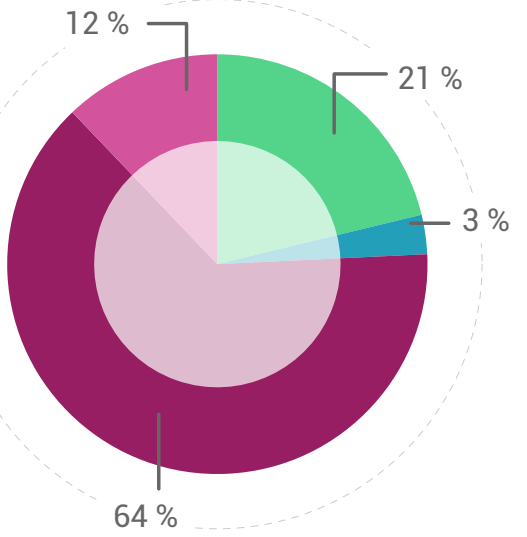

Figura 3. Actividades que debe realizar en su dependencia para garantizar el éxito en la adopción de las NICSP.

El $64 \%$ de los encuestados son conscientes de las actividades que deben realizar para garantizar el éxito de la adopción de las NICSP en sus dependencias, tales como capacitarse, conocer la norma de implementación y actualizar el software para ello. Un $21 \%$ hace especial referencia a la necesidad de capacitación, un $12 \%$ no sabe o no responde y solo un $3 \%$ manifiesta que el conocimiento de la norma es una necesidad (Figura 3).

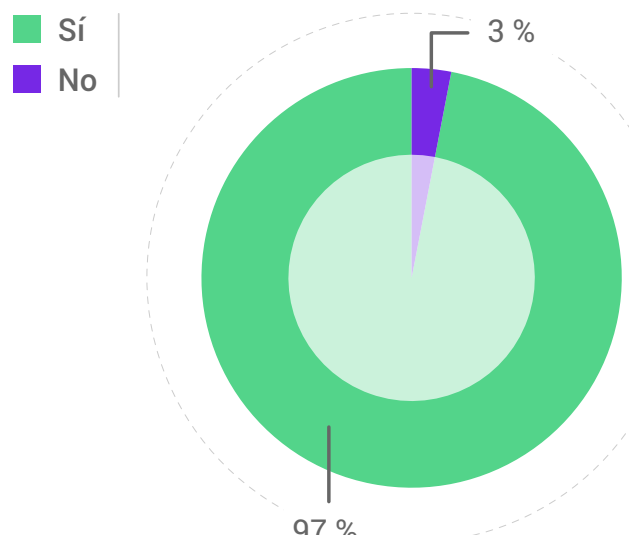

Figura 4. Existencia de manuales que hablen de los cambios que exigen las NICSP.

El $97 \%$ de los encuestados dice no contar con manuales que hablen de los cambios que exigen las NICSP, y solo un 3 \% manifestó que sí cuentan con dichos manuales, lo que deja entrever la poca planeación que ha tenido el proceso de apropiación de las NICSP en la población analizada. 
Activos

Todas las anteriores

NS/NC

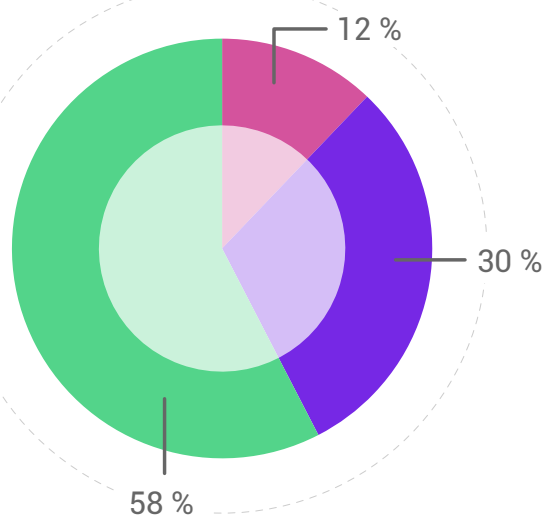

Figura 5. Conocimientos de los funcionarios de los colegios sobre las cuentas más afectadas con la incorporación de las NICSP en el régimen de contabilidad pública.

Solo el $30 \%$ de las instituciones conocen las cuentas de la información contable que serán más afectadas con la incorporación de las NICSP en el régimen de contabilidad pública, dentro de las cuales se encuentran los activos, los pasivos, el patrimonio y las inversiones; el $58 \%$ no sabe $o$ no responde, y solo el $12 \%$ afirma conocer solamente la afectación que tendrá las cuentas de los activos (Figura 5).

De acuerdo con la Contaduría General de la Nación, la finalidad de las NICSP es suministrar una información transparente y oportuna en los estados financieros, ampliando la calidad de la información presentada por todo el personal vinculado para la rendición de cuentas de los recursos públicos y para la toma de decisiones; el concepto anterior mencionado lo conocen el $22 \%$ de los encuestados y solo el $14 \%$ conocen los cambios que la entidad pública debe ejecutar, donde es de especial importancia realizar los ajustes necesarios a los sistemas informáticos propios, para empezar a registrar las operaciones diarias en aplicación de la nueva norma, para lo cual también se deben saldar aquellas cuentas que se eliminarán del plan de cuentas al término del actual periodo contable.

Se pudo reflejar en el análisis de la información que siendo solo el $20 \%$ de los encuestados contadores públicos no se ve una coherencia en la respuesta en el perfil del contador, donde se muestra que el $76 \%$ tiene el perfil para la implementación de las NICSP; sin embargo, el $88 \%$ conoce las necesidades de su personal contable para la implementación de las NICSP entre ellas: conocimiento y preparación de información de acuerdo con estándares internacionales de información financiera, un sistema contable, definición de criterios técnicos contables aplicables al sector público educativo y homogeneidad en las políticas contables. 


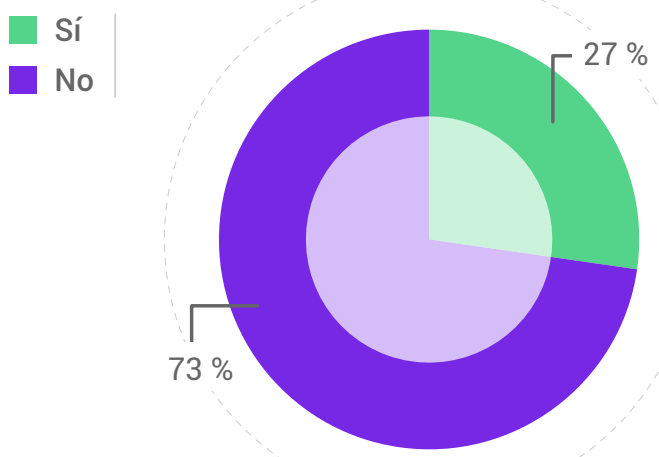

Figura 6. Cambios que traerá la implementación de las NICSP en los inventarios de la propiedad planta y equipo de las instituciones educativas.

El $73 \%$ de los funcionarios no conoce los cambios que traerá consigo la implementación de las NICSP en materia de los inventarios de la propiedad planta y equipo de las instituciones educativas, solo el $27 \%$ dice tener un conocimiento al respecto (Figura 6), y el $79 \%$ concuerda en que con la implementación de políticas se mejorará el proceso de registro, manejo y custodia de bienes.

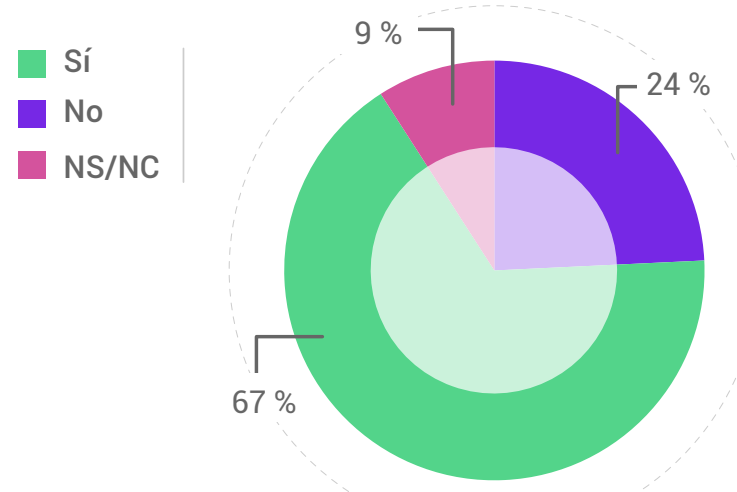

Figura 7. Recursos humanos y económicos para la implementación de las políticas contables en el manejo de los inventarios y procesos de depuración contable.

El $67 \%$ de las instituciones educativas no cuenta con los recursos humanos y económicos para la implementación de las políticas contables en el manejo de inventarios y proceso de depuración contable, solo el 24 \% manifestó si contar con los recursos humanos y económicos para tal fin (Figura 7). 


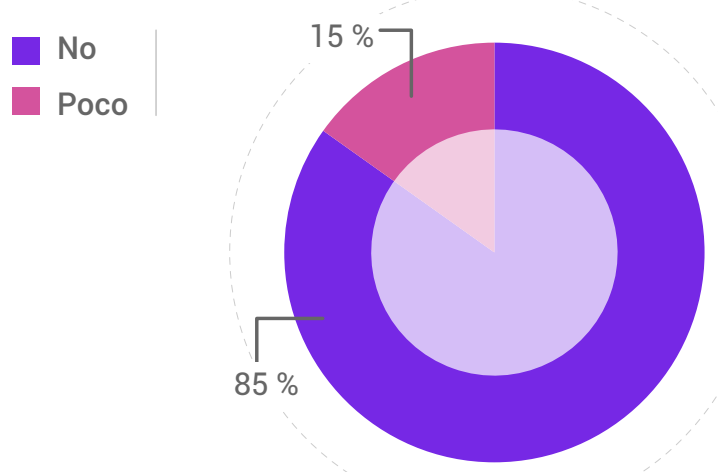

Figura 8. Capacitación del ente territorial a las instituciones educativas para la implementación de las NICSP.

Las instituciones desconocen en su gran mayoría las políticas contables que se deben implementar en materia de propiedad planta y equipo y no han sido capacitados por el ente territorial para la implementación de las NICSP y solo el $15 \%$ de las instituciones educativas cuentan con el personal capacitado para la implementación de las NICSP (Figura 8).

Los encuestados manifiestan en un $85 \%$ que el ente territorial no se ha preocupado por brindar capacitaciones para la implementación de las normas internacionales de contabilidad para el sector público, frente a un $15 \%$ que aducen haber recibido algún tipo de orientación sobre el particular.

\section{Discusión}

\section{Antecedentes}

El proceso de actualización de la regulación contable en Colombia se puede considerar un proceso permanente y en evolución. El primer Plan General de Contabilidad Pública fue expedido mediante la resolución 4444 de 1995 por parte del Ministerio de Hacienda. Posteriormente, en el año 2000 se creó la CGN para ajustar la regulación contable a la realidad de las entidades.

En el año 2004 se inició el proyecto tendiente a la armonización de las NICSP que habían sido emitidas en el año 2000. Como resultado del proyecto, en el año 2007 se expidieron las resoluciones 354, 355 y 356 mediante los cuales la CGN adoptó el Régimen de Contabilidad Pública, el Plan General de Contabilidad Pública y el Manual de Procedimientos, respectivamente. 
Ya en el año 2009, se emprendió otro nuevo proyecto con el fin de actualizar y armonizar el Régimen de Contaduría Pública con estándares internacionales de Contabilidad e incorporar criterios de las Normas Internacionales de Información Financiera para empresas estatales.

Producto de esta última iniciativa la CGN expidió la resolución 743 (2013) mediante la cual incorporó en el Régimen de Contaduría Pública el nuevo marco normativo aplicable a empresas propiedad del estado, cuyos ámbitos de aplicación, cronograma y vigencias se encuentran consignados en la resolución 533 de 2015. También expidió la Resolución No. 414 (2014b) y sus modificaciones (Instructivo 002 (2014a), Resolución 139 (2015a), Resolución 633 (2015), Resolución 466 (2016) ), mediante la cual se incorporó el Marco Conceptual para la Preparación y Presentación de la Información Financiera (Contaduría General de la Nación \& Ministerio de Hacienda y Crédito Público, 2014a) y las Normas para el Reconocimiento, Medición, Revelación y Presentación de los Hechos Económicos (Contaduría General de la Nación \& Ministerio de Hacienda y Crédito Público, 2014b).

La Resolución 192 del 05 de mayo de 2016, incorpora en la estructura del RCP el elemento procedimientos transversales y el 19 de agosto de 2016 fueron expedidas las resoluciones 468 por medio de la cual se modifica el catálogo general de cuentas del marco normativo para entidades del gobierno; 469 por la cual incorpora al Marco Normativo para Entidades de Gobierno, el Procedimiento contable para el registro de los hechos económicos relacionados con la movilización de activos y la 470 por la cual incorpora al Marco Normativo para Entidades de Gobierno, el procedimiento contable para el registro de los recursos del sistema general de regalías. Adicionalmente, la
CGN ha venido desarrollando eventos con participación de funcionarios miembros de instituciones homólogas de otros países, con el fin de compartir experiencias sobre la aplicación e implementación de las NICSP.

Todo lo anterior muestra un escenario de permanentes cambios en el cual el capital humano de los funcionarios que llevan a cabo estas tareas contables juega un papel primordial. Por tanto, es necesario que las entidades territoriales estén en capacidad de dar respuesta a las transformaciones que exige la normativa y eso sugiere la necesidad de una permanente capacitación de sus funcionarios, de manera que los procesos sean hechos no solo correctamente, sino también de manera eficiente y concorde con las políticas de transparencia que las entidades territoriales deben demostrar.

\section{Implementación de las NICSP en el Régimen de Contabilidad Pública de Colombia}

Las Normas Internacionales de Contabilidad del Sector Público, cuya sigla es NICSP, son un conjunto de normas esenciales para registrar, medir y revelar, los hechos económicos de las entidades que se encuentran bajo el Régimen de Contabilidad Pública (RCP), su objetivo principal es suministrar una información transparente y oportuna en los estados financieros, ampliando la calidad de la información presentada por todo el personal vinculado para la rendición de cuentas de los recursos públicos y para la toma de decisiones, de manera que la Contraloría General de la Republica (CGR) está desarrollando todo el proceso necesario para dicha convergencia, siendo a la vez el responsable de distribuir las normas y definir los procesos contables básicos de todas 
las instituciones públicas que administran los recursos del País (Contaduría General de la Nación decreto Nro. 2615, 2014; Resolución Nro.533, 2015, Resolución Nro. 414, 2014).

De igual manera las NICSP son importantes porque aumentan la calidad y el detalle de la información contable, proveen un límite mínimo adecuado y bien entendido para la calidad de la información financiera de los gobiernos y otras entidades del sector público; son medidas que harán mucho más fácil la comparación de información entre los gobiernos, puesto que se habrá fortalecido la transparencia en la rendición de cuentas de los recursos públicos.

Cabe señalar que la contabilidad del sector público está bajo el RCP, mediante la resolución Nro. 354 de 2007, el cual contiene la regulación contable pública de tipo general y específico y está conformado por el plan general de contabilidad pública, por el manual de procedimientos y toda la doctrina contable pública, sin embargo, se encuentran marcadas diferencias entre la información de los Estados Financieros presentados bajo el RCP y la información de los Estados Financieros presentados bajo las NICSP; algunas de las diferencias de ambos modelos radica cuando se habla de preparación de la información contable bajo el concepto del negocio en marcha y del momento en el que se deben realizar los ajustes, así:

En las NICSP se reportan los hechos ocurridos después de la fecha de presentación, mientras que en el RCP se reportan los hechos posteriores al cierre. En los hechos que implican ajuste las NICSP usa ejemplos, pero no criterios de materialidad, mientras que el RCP usa solo hechos ciertos de materialidad. En los hechos económicos que no implican ajustes las NICSP usa ejemplos generales y el RCP usa caracterizaciones. Ahora bien, así como se encuentran diferencias también se encuentran semejanzas entre dichos modelos, ambos indican que no se deben ajustar hechos ocurridos después del periodo; así también en los dos modelos se requiere la revelación de hechos materiales (Comparativos entre las Normas Internacionales de Contabilidad del Sector Público NICSP y el Régimen de Contabilidad Pública RCP, 2011).

Es importante resaltar que los elementos de las NICSP, que se van a incorporar dentro del RCP, generarán gran impacto en la situación financiera de cada una de las entidades públicas, lo anterior debido al retiro de algunos activos y la incorporación de pasivos a partir de las definiciones de activo y pasivo del nuevo marco normativo, también en el reconocimiento de los activos a partir del criterio de control como lo determina las NICSP y no del criterio de propiedad como se ha venido haciendo bajo el RCP. Este proceso generará cambios en las cifras de algunas cuentas como activo, pasivo y patrimonio que para algunas entidades será más impactante que para otras, puesto que los elementos que se incorporarán al RCP serán de criterios transversales de reconocimiento, como es la asociación del hecho económico con los elementos de los estados financieros, con una medición confiable, donde haya probabilidad de entrada y salida de flujos de efectivo; asimismo se reclasificarán algunas partidas como son propiedad planta y equipo, a propiedades de inversión o activos biológicos; como también los bienes adquiridos por medio de leasing financieros a propiedad planta y equipo; además, se incorporará al RCP elementos de instrucciones, con el fin de evaluar indicios de deterioro en cada una de las entidades públicas (Estado actual y prospectiva del proceso de convergencia en el sector público, Contaduría General de Nación). 
Ahora bien, estos elementos deben ser ingresados al RCP debido a la preocupación sobre la calidad de la información financiera presentada por muchos gobiernos y sus organismos, ya que se presentaba una información de escasa confiabilidad, de baja calidad y generalmente pobre en justificación y soporte. Estas falencias determinan muchas veces el poco éxito de los objetivos gubernamentales, las deficiencias en la toma de decisiones económicas y la inadecuada rendición de cuentas que conlleva a un incremento de la corrupción; es por esto que al ingresar dichos elementos de las NICSP al RCP se busca hacer responsables a los gobiernos, sus organismos y sus funcionarios para que haya una buena gobernabilidad y transparencia fiscal como condición necesaria para un debido desarrollo en las rendiciones de cuentas.

Los elementos que conlleva implementar este proceso comprenden un conjunto de acciones, tareas y actividades secuenciales que todas las entidades públicas deben iniciar; estas actividades tienen que ver con el análisis de las normas contables que le serán asignadas a cada área, como también detectar las necesidades de capacitación y la evaluación del impacto de dicha implementación sobre cada proceso.

En consecuencia con lo anterior y mediante la Resolución de la Contraloría General de la República Nro. 16 de 2015 NICSP-CGR Normativa del Sistema de Contabilidad General de la Nación para el Sector Público, para el año 2018 se espera que todos los Estados Financieros del sector público estén presentados bajo las NICSP; para esto se necesita de un equipo líder que lleve a cabo todas las acciones necesarias para sacar adelante este objetivo. Este equipo debe estar conformado por distintos perfiles, no únicamente contadores públicos, sino también profesionales encargados de la administración, el área de sistemas y la tecnología; asimismo, el liderazgo debe ir al frente de un alto mando directivo con el fin de buscar un complemento desde varias matices y puntos de vista.

Para la implementación de las NICSP cada área debe tener un encargado y un plan operativo. Cada actividad se debe calificar por su relevancia, cuáles aplican plenamente, parcialmente o cuáles definitivamente no aplican para el proceso. En la planificación se deben adoptar las principales decisiones estratégicas, elaborar el plan de implementación institucional y desarrollar las actividades que permitirán la posterior implementación de la normativa; en la implementación se incluyen los cambios que la Entidad Pública debe ejecutar y es de especial importancia realizar los ajustes necesarios a los sistemas informáticos propios para empezar a registrar las operaciones diarias en aplicación de la nueva norma, para lo cual también se debe saldar aquellas cuentas que se eliminarán del plan de cuentas al término del actual periodo contable; finalmente, para la sostenibilidad se debe tener como objetivo generar las acciones de mejora continua del proceso de implementación, con respecto a las nuevas normas complementarias que emitirá en los próximos años el Consejo de Normas Internacionales de Contabilidad del Sector Público, para poder desarrollar las acciones de mejora en la emisión de reportes financieros para que estén en condiciones de ser empleados por todos los usuarios vinculados al proceso; esta necesidad es sugerida por la Contraloría General de la Nación; apoyada por la resolución Nro. 16 (2015).

Por lo anteriormente expuesto, se puede mencionar que la capacitación para los funcionarios encargados del área contable de las instituciones públicas de Manizales es un 
tema de vital importancia al que se le debe establecer instrumentos que ayuden a identificar el problema, recopilar los antecedentes que lo ocasionan, estableciendo la solución para dicha implementación.

El contenido de dichas capacitaciones debe presentar los retos planteados para el proceso de implementación de las NICSP, las acciones estratégicas definidas por la norma, hacer una adecuada planificación estratégica que garantice los aspectos de interés de la norma en función de la situación actual, como también una adecuada planificación de los alcances, el presupuesto y el tiempo de ejecución, para así garantizar un exitoso proceso de implementación que, además, contenga un procedimiento de soporte y mejora continua para brindar el mantenimiento y actualización necesaria que la norma exige a lo largo del tiempo.

\section{Desafíos del personal profesional y auxiliar contable ante la implementación de las NICSP}

La convergencia a las Normas internacionales de Contabilidad del Sector Público NICSP es una realidad hoy a nivel global, no por un capricho del Gobierno colombiano, sino por la necesidad de homogenizar el registro de todos los recursos y obligaciones del sector público y así conformar un sistema integral único y uniforme.

Se nota que las entidades públicas aún no se encuentran preparadas para enfrentar el reto de la implementación y aplicación de las NICSP, puesto que muchas de ellas desconocen el verdadero impacto en los costos de formación, tecnología, recursos y consultoría que implica este proceso. Es por esto que una de las personas que hacen parte fundamental para este proceso son los contadores públicos y auxiliares contables. La ley 43 (1990) en su capítulo primero menciona que:

En Colombia se puede decir que el contador público es la persona natural que mediante previa inscripción ante la Junta Central de Contadores, y después de acreditar su competencia profesional (Título universitario), está facultado por la ley para dar fe pública respecto de los hechos conocidos por él y propios del ámbito de su profesión, lo mismo que dictaminar sobre la información económica y financiera, realizar las actividades relacionadas con la ciencia contable, tributaria, los sistemas de información de la empresa, las finanzas, los costos entre otros (p. 1).

Según Aragón (2009) en su artículo «El nuevo papel del contador frente a la transición hacia las Normas internacionales de información financiera» establece que:

El contador público es el ser crítico e imparcial de la entidad pública, de él depende que la información financiera que se presenta sea transparente y además que las decisiones que se tomen sean las más convenientes y acordes a la realidad del ente (p. 7).

En este sentido, el perfil del contador público y de los auxiliares contables debe ir mucho más allá, con el fin de que puedan apoyar de forma estratégica y operativa la implementación de las NICSP en cada una de las entidades estatales. Es por esto que se debe:

Cambiar la visión que se tiene frente a la formación de los contadores públicos; la nueva imagen del profesional debe ser integral, con espíritu de liderazgo, competitividad, sabiduría en la toma de decisiones, manejo y dominio de las Normas Internacionales de Información Contable en el Sector Público. Para desempeñar este nuevo rol 
el contador público necesita: capacidad de comprender y aplicar con celeridad los nuevos esquemas y tecnologías; leer y saber redactar; estudiar las normas tributarias; concientizarse del papel tan importante que juega en la economía; estudiar profundamente las Normas Internacionales de Información Contable en el Sector Público NICSP y sus cambios; aplicar el código de ética profesional (Aragón, 2009, p. 7).

Para el Instituto Nacional de Contadores Públicos (INCP) (2016) el reto y desafío personal involucrado en la implementación de las NICSP, es muy grande e indispensable, a continuación, se establece varios criterios que debe tener los contadores públicos y auxiliares contables para este tema.

El primer criterio establece el conocimiento que el personal involucrado debe tener sobre la Norma Internacional en el Sector Público, debe de ser un conocimiento crítico y analítico en el cual se pueda emitir juicios acordes a la convergencia, transición y aplicación de la Norma. Como segundo lineamiento se plantea el asesoramiento de expertos bajo NIIF y NICSP del personal. Este asesoramiento acompaña el proceso de aplicación e implementación de la NICSP en las entidades y del mismo modo asegura que el proceso se lleve de la mejor manera.

Se tiene como tercer criterio la sostenibilidad del proceso de Convergencia de las Normas Internacionales en el Sector Público; es indispensable contar con la disposición y actitud de cambio del personal, dado que de este depende la sostenibilidad de la Aplicación de las NICSP cuando ya no se cuente con el acompañamiento de los asesores expertos.

De esta forma el cuarto lineamiento que se tiene, según el Instituto Nacional de contadores públicos (INCP) (2016), es el siguiente:
Los impactos obtenidos a nivel de recursos tecnológicos y de procesos; se debe asegurar que los sistemas contables soporten las nuevas políticas y el asegurar que el gobierno haya pensado en la adaptación del sistema de información SIIF y de manera integral, de lo contrario se tendrán que desarrollar otras aplicaciones para poder hacer del proceso algo más automático y menos dispendioso (párr. 15).

Teniendo en cuenta lo antes relacionado, se deben realizar algunas acciones de forma muy detallada para proceder con la convergencia e implementación de las NICSP, siendo una de ellas la depuración de las cuentas. Estas cuentas se deben analizar de forma individual para de este modo hacer el debido traslado al balance general y saldos, con el fin de tener la apertura de las cuentas y comenzar con la implementación del sistema contable bajo NICSP. Otra de las actividades de mayor relevancia es realizar las políticas contables de las entidades del Estado. Se debe aplicar las políticas contables que realmente las entidades necesitan (Peñuela, 2016).

De esta forma, la Contaduría General de la Nación (CGN) como entidad reguladora de la contabilidad de todos los organismos del Estado, en su documento «Estrategia de convergencia de la regulación contable pública hacia Normas Internacionales de Información Financiera y Normas Internacionales de Contabilidad del Sector Público» establece los lineamientos, bondades y modernización que trae la implementación de NICSP (Contaduría General de la Nación de Colombia, 2013).

Teniendo en cuenta lo anterior, uno de los cambios relevantes para las instituciones del Estado es el manejo de las propiedades planta y equipo (PPyE). De acuerdo con el decreto 2650 (1993): 
Las propiedades, planta y equipo deben registrarse al costo histórico, del cual forman parte los costos directos e indirectos causados hasta el momento en que el activo se encuentre en condiciones de utilización o en condiciones de puesta en marcha o enajenación, tales como los de ingeniería, supervisión, impuestos, corrección monetaria proveniente de la unidad del poder adquisitivo contante - UPAC (hoy UVR) e intereses (párr. 2).

\section{Según la NICSP-17:}

Establece que la Propiedad, planta y equipo son en general la mayor parte de los activos de una entidad, y, por lo tanto, resultan significativos en el contexto de su situación financiera. Además, la determinación de si un gasto representa un activo o un gasto puede tener un efecto importante en el resultado neto (ahorro o desahorro) de las actividades operativas de una entidad; para poder dar reconocimiento a un bien de PPyE primero se debe determinar si un rubro concreto cumple con el primer criterio de reconocimiento como activo, debe evaluarse el grado de certidumbre relativo a los flujos de beneficios económicos futuros o los potenciales servicios, a partir de la evidencia disponible en el momento del reconocimiento inicial; El segundo de los criterios para el reconocimiento como activo se satisface de forma inmediata, puesto que la transacción que pone de manifiesto la compra del activo relaciona su costo; A fin de identificar qué constituye una partida (o rubro) separada, dentro de los elementos componentes de la Propiedad, planta y equipo, se precisa la realización de juicios para la aplicación de los criterios en la definición de las circunstancias o los tipos de entidades específicas; Todo elemento de la Propiedad, planta y equipo, que cumpla las condiciones para ser reconocido como un activo debe ser medido, inicialmente, por su costo; cuando un activo se adquiere por un costo nulo o insignificante, su costo es su valor razonable en la fecha de la adquisición (NICSP-17, s.f, p. 486).

\section{Conclusiones}

- Los elementos de las NICSP que se van a incorporar dentro del RCP generarán gran impacto en la situación financiera de cada una de las entidades públicas, lo anterior debido a la reclasificación de algunos activos y la incorporación de pasivos, a partir de las definiciones de activo y pasivo del nuevo marco normativo, también en el reconocimiento de los activos a partir del criterio de control como lo determina las NICSP y no del criterio de propiedad como se ha venido haciendo bajo el RCP.

En Colombia bajo la supervisión de la CGN se busca generar garantías encaminadas a la información financiera, pues debe ser de alta calidad, útil, confiable, oportuna ajustada a los requerimientos de las normas internacionales, que sirva para la toma de decisiones, mejorando la transparencia, la rendición de cuentas y desarrollando un control financiero sobre las actividades de las entidades públicas.

- Se evidencia que las entidades públicas aún no se encuentran preparadas para enfrentar el reto de la implementación y aplicación de las NICSP, puesto que muchas de ellas desconocen el verdadero impacto en los costos de formación, tecnología, recursos y consultoría que implica este proceso.

- De acuerdo con los resultados obtenidos por el trabajo de campo realizado en el presente artículo, se puede evidenciar la necesidad de implementar programas de capacitación que direccionen a las instituciones educativas hacia la implementación de las NICSP, pero queda para reflexión de los lectores que las mismas no cuentan con el personal idóneo a nivel profesional para 
lograr tal objetivo, convirtiéndose en una gran amenaza para el bienestar financiero de la entidad y las posibles sanciones a las cuales puede acarrear.

- Es importante resaltar la negativa en el trabajo de campo por parte de las instituciones educativas, puesto que solo el $48.6 \%$ respondieron el instrumento de investigación, lo que indica que puede existir una parcialidad en la información recolectada y analizada, pudiendo incidir en las necesidades reales de formación del personal contable de las instituciones públicas de educación básica y media de la ciudad de Manizales, en relación a la implementación de las NICSP para el año 2016.

\section{Referencias}

- Aragón, J. (2014). El Nuevo Papel Del Contador Frente A La Transición Hacia Las Normas Internacionales de información financiera. Recuperado de https://goo.gl/NiqB4N

- Contaduría General de la Nación. (2013). Estrategias de convergencia entre las NIIF y NICSP. Recuperado de https://goo.gl/7Gs9TX

- Contaduría General de la Nación. Resolución 743 (2013). Recuperado de https:// goo.gl/pGdja1

- Contaduría General de la Nación. Instructivo 002 (2014). Recuperado de https://goo. $\mathrm{gl} / \mathrm{JGo3rs}$

- Contaduría General de la Nación. Resolución 414 (2014). Recuperado de https:// goo.gl/bQScBs
Contaduría General de la Nación. Resolución 139 (2015). Recuperado de https:// goo.gl/DPVUKa

- Contaduría General de la Nación. Resolución 533 (2015). Recuperado de https:// goo.gl/eqAqWW

- Contaduría General de la Nación. Resolución 633 (2015). Recuperado de https:// goo.gl/9EruQN

- Contaduría General de la Nación. Resolución 466 (2016). Recuperado de https:// goo.gl/1KRSjo

- Contaduría General de la Nación, y Ministerio de Hacienda y Crédito Público (Eds.). (2013). Estrategia de convergencia de la regulación contable pública hacia normas internacionales de información financiera (NIIF) y normas internacionales de contabilidad del sector público (NICSP). Santafé de Bogotá: Contaduría General de la Nación.

- Contaduría General de la Nación, \& Ministerio de Hacienda y Crédito Público. (2014a). Marco Conceptual para la Preparación y Presentación de Información Financiera. De las Empresas que no Cotizan en el Mercado de Valores, y que no Captan ni Administran Ahorro del Público. Recuperado de https:// goo.gl/uUGiMc

- Contraloría General de la República de Chile. (2015). Guía para la implementación institucional de las NICSP-CGR. Recuperado de https://goo.gl/LuVimD

Decreto 2650. (1993). Por el cual se modifica el Plan Único de Cuentas para Comerciantes. Recuperado de https://goo.gl/4CoqRE 
- Hernández, R., Fernández, C., \& Baptista, P. (2010). Metodología de la investigación (5a ed). México, D.F: McGraw-Hill.

- IFAC. (2009). Las Normas Internacionales de Contabilidad para el Sector Público Revisadas ya se Encuentran Disponibles en español. Recuperado de https://goo.gl/SR1q5d

- Ley 43. (1990). Reglamentaria de la profesión de contador público y se dictan otras disposiciones. Recuperado de https://goo. gl/EkxLMh
- Ministerio de Educación Nacional. Decreto 4791 (2008). Recuperado de https://goo. $\mathrm{gl} / \mathrm{xBa} 3 \mathrm{Yd}$

- NICSP- 17, (s.f). Propiedad Planta y Equipo. Recuperado de https://goo.gl/vmrvW3

- Peñuela, A. (2016). Normas internacionales de Contabilidad de Sector Público, su aplicación y los desafíos en Colombia. Recuperado de https://goo.gl/Dfm $1 \mathrm{eC}$ 